\title{
Produtividade de banana submetida a diferentes níveis de salinidade da água de irrigação: Segundo ciclo ${ }^{1}$
}

\author{
Ancélio R. de O. Gondim², José F. de Medeiros ${ }^{3}$, Gilcimar A. do Carmo ${ }^{3}$, Francisco H. F. Pereira ${ }^{2}$, Hans R. Gheyi ${ }^{4}$ \& José C. Tavares ${ }^{3}$
}

\section{RESUMO}

Tendo em vista a grandeza dos problemas de salinidade na região Nordeste e a inexistência de informações de como utilizar as águas salinas adequadamente na cultura de bananeira, objetivou-se, com este trabalho verificar os efeitos de diferentes níveis de salinidade da água de irrigação sobre a produtividade de banana (Musa spp.), no segundo ciclo. Utilizou-se o delineamento experimental em blocos casualizados, no esquema de parcelas subdivididas, com quatro repetições. As parcelas foram constituídas de três níveis de salinidade da água de irrigação $(1,7,2,8$ e 4,0 dS m-1) e as subparcelas por duas cultivares de banana (Pacovan - AAB e Marmelo - ABB). Verificou-se reduções de 21,27, 17,90, $19,67,5,89,1,93$ e $17,90 \%$, respectivamente para a massa total das pencas, massa do cacho, massa média dos frutos, número de pencas por cacho, número de frutos por cacho e produtividade, com o aumento na condutividade elétrica da água de irrigação de 1,7 para 4,0 dS m-1. O menor nível de salinidade da água de irrigação estudado $\left(1,7 \mathrm{dS} \mathrm{m}^{-1}\right)$, proporcionou maior produtividade. A cultivar 'Marmelo' mostrou maior potencial produtivo em relação à 'Pacovan'. O efeito da salinidade nas cultivares foi semelhante.

Palavras-chave: bananeira, condutividade elétrica, rendimento

\section{Productivity of banana submitted to different levels of salinity of irrigation water: Second cycle}

\begin{abstract}
Keeping in view the salinity problems in the Northeast region and the inexistence of information of how to use the saline waters appropriately, the objective of this work was to verify the effects of different levels of irrigation water salinity on the productivity of banana (Musa spp.), in the second cycle. A randomized block design was used, with four replications in split-plot. The plots were constituted by three levels of salinity of irrigation water $\left(1.7,2.8\right.$ and $\left.4.0 \mathrm{dS} \mathrm{m}^{-1}\right)$ and the subplot by two varieties of banana (Pacovan - AAB and Marmelo - ABB). Reductions of 21.27, 17.90, 19.67, $5.89,1.93$ and $17.90 \%$ were verified, respectively, in the total mass of the bunches, mass of the bunch, mean mass of the fruits, number of bunches per bunch, number of fruits per bunch and productivity, with the increase in the electrical conductivity of the irrigation water from 1.7 to $4.0 \mathrm{dS} \mathrm{m}^{-1}$. The lowest level of salinity of the irrigation water studied $\left(1.7 \mathrm{dS} \mathrm{m}^{-1}\right)$, promoted satisfactory production. The variety Marmelo showed higher productive potential in relationship to Pacovan. The effect of salinity on the cultivars was similar.
\end{abstract}

Key words: banana, electrical conductivity, yield 


\section{INTRODUÇÃO}

A diminuição na disponibilidade de água de boa qualidade para irrigação se deve à distribuição para outros setores (urbano e indústria), especialmente em regiões áridas e semiáridas, como a Região Nordeste, que resulta no uso intensivo de água de baixa qualidade (salina) para satisfazer a demanda crescente na irrigação. O uso de tal água causa um aumento na salinidade do solo que pode ter efeitos negativos no crescimento e rendimento da cultura. (FAO, 1990).

A salinização das áreas irrigadas é notória e, no Nordeste do Brasil, tem-se constatado este problema praticamente em todos os grandes perímetros irrigados. Normalmente, a salinidade em áreas irrigadas é conseqüência de uma pobre drenagem do solo, aplicação insuficiente de água e o uso da água salina, associado ao manejo do sistema solo-água-planta (Medeiros et al., 1993). A utilização da irrigação no Nordeste brasileiro, onde a cultura da banana é muito explorada, tem proporcionado sérios problemas de salinização do solo (Bernardo, 1995). O elemento predominante na maioria dos solos salinos é o sódio, facilmente absorvido por estar presente em maior quantidade que os outros elementos (Marschner, 1995). A salinidade causa redução na absorção de água e nutrientes, no crescimento da planta, limita a fotossíntese e, deste modo, o acúmulo de massa seca e a produtividade das culturas (Drew et al., 1990).

A má utilização da água pode aumentar o $\mathrm{pH}$ e os teores de Ca no solo o que pode promover desequilíbrio de nutrientes na bananeira, que é muito sensível ao desequilíbrio entre Ca, K e Mg (Silva et al., 1999). Costa \& Gheyi (1984) avaliaram a qualidade das águas da região de Catolé do Rocha, (PB), e verificaram que grande parte das águas apresentava elevadas concentrações de $\mathrm{HCO}_{3}, \mathrm{Ca}^{2+}, \mathrm{Mg}^{2+}$ e $\mathrm{Na}^{+}$. Resultados semelhantes foram encontrados por Maia et al. (1997) na avaliação da qualidade das águas de poços tubulares, de rios e de açudes da Chapada do Apodi, (RN). A salinização pode causar, no solo, entre outros processos, mudanças nas suas propriedades químicas e físicas (Bernardo, 1995).

A bananeira é sensível à salinidade e, para seu bom desenvolvimento vegetativo, requer valores de condutividade elétrica da água de irrigação menores que $1,00 \mathrm{dS} \mathrm{m} \mathrm{m}^{-1}$, para alcançar boa produtividade, porém, com o aumento da condutividade para $6,00 \mathrm{dS} \mathrm{m}^{-1}$ na cultivar 'Nanica', provocou decréscimo de $40 \%$ na produtividade, além de atrasar a emissão do cacho em, aproximadamente, um mês (Abreu et. al., 1982).

A avaliação da produtividade de banana sob condições salinas é muito limitada. Diversos resultados são atribuídos ao efeito do estresse salino sobre o crescimento das plantas, como os trabalhos desenvolvidos em Mossoró, RN (Carmo et al., 2003); em Recife, PE (Ulisses et al., 2000); em Viçosa, MG (Neves et al., 2002) e Campina Grande, PB (Araújo Filho et al.,1995; Santos \& Gheyi, 1994).

A redução geral no crescimento da planta e produtividade das culturas, tem-se tornado comportamento corriqueiro verificado em diversos trabalhos, quando as plantas são submetidas ao estresse salino. Este comportamento é atribuído à redução no potencial hídrico da solução do solo gerado pelo efeito osmótico dos íons, adicionados em grandes quantidades pelo uso continuado de água salina, como verificado por Gondim et al. (2002), dificultando a absorção de água pelas raízes das plantas de banana. Como a água é um dos fatores essenciais para a expansão celular, sua limitação implica em menor crescimento de células e tecidos, também menor incremento em altura da planta, número de folhas e área foliar da bananeira (Carmo et al., 2003), e, conseqüentemente, menor produtividade. Outro fator a ser considerado é a diminuição nas taxas fotossintéticas sob condições de estresse salino, como relatado em diversos pesquisadores (Meloni et al., 2001; Pereira et al., 2004). A diminuição da fotossíntese sob condições de estresse salino tem sido atribuída ao fechamento dos estômatos, para evitar a perda excessiva de água, absorvida de maneira limitada pelas raízes devido à redução no potencial osmótico na solução do solo. Em virtude do fechamento estomático, a aquisição e fixação de $\mathrm{CO}_{2}$ no processo fotossintético são reduzidas, comprometendo o crescimento e a produtividade das culturas.

Ante a carência de resultados de pesquisa com a cultura da banana este trabalho teve o objetivo de estudar os efeitos de diferentes níveis de salinidade da água de irrigação sobre a produtividade de duas cultivares de banana, 'Marmelo' e 'Pacovan', no segundo ciclo de produção.

\section{MATERIAL E MÉTODOS}

O experimento foi desenvolvido em área do Parque ZôoBotânico da Escola Superior de Agricultura de Mossoró, RN, ESAM, no período de julho/2000 a maio/2001, segundo ciclo, em solo classificado como Argissolo Vermelho Amarelo, textura franco-argilo-arenosa (EMBRAPA, 1999). O município de Mossoró está situado na região semi-árida do Nordeste brasileiro, no Estado do Rio Grande do Norte ( $5^{\circ} 11^{\prime} \mathrm{S}, 37^{\circ} 20^{\prime} \mathrm{W}$ e altitude de $18 \mathrm{~m}$ ). As análises químicas de amostras do solo foram realizadas no Laboratório de Solos da ESAM (Tabela 1). As condições climáticas médias mensais registradas durante o tempo de condução do experimento, foram: temperatura mínima de 16,5 a $20,7{ }^{\circ} \mathrm{C}$; média de 25,7 a $29,9{ }^{\circ} \mathrm{C}$ e máxima de 34,1 a $37,4{ }^{\circ} \mathrm{C}$; umidade relativa de 66 a $86 \%$ e precipitação pluvial de 0,0 a $63,5 \mathrm{~mm}^{\text {mês }}{ }^{-1}$. O relevo é considerado plano a suave ondulado.

O delineamento experimental utilizado foi em blocos casualizados, com quatro repetições, no qual se empregou o esquema de parcelas subdivididas. Nas parcelas foi aplicada água de irrigação com três níveis de salinidade (1,7; 2,8 e 4,0 dS m $\mathrm{m}^{-1}$ ) e nas subparcelas foram utilizadas duas cultivares de banana (Pacovan - AAB e Marmelo - ABB). Cada

Tabela 1. Características químicas do solo da área experimental

\begin{tabular}{|c|c|c|c|c|c|c|c|c|}
\hline \multirow{2}{*}{ PROF } & $\mathrm{pH}$ & $\mathrm{Ca}^{2+}$ & $\mathrm{Mg}^{2+}$ & $\mathbf{K}^{+}$ & $\mathrm{Na}^{+}$ & $\mathrm{Al}^{3+}$ & \multirow{2}{*}{$\begin{array}{c}P \\
\left(\mathrm{mg} \mathrm{dm}^{3}\right)\end{array}$} & \multirow{2}{*}{$\begin{array}{l}\text { CEes } \\
\left(\mathrm{dS} \mathrm{m}^{-1} \text { ) }\right.\end{array}$} \\
\hline & $\begin{array}{c}(1.2, \mathrm{O}) \\
\mathrm{H}_{2} \mathrm{O}\end{array}$ & \multicolumn{5}{|c|}{$\left(\mathrm{cmol}_{\mathrm{c}} \mathrm{dm}^{-3}\right)$} & & \\
\hline $0-40$ & 6,6 & 4,5 & 4,5 & 0,36 & 0,26 & 0,00 & 90 & 2,7 \\
\hline
\end{tabular}

R. Bras. Eng. Agríc. Ambiental, v.10, n.1, p.38-42, 2006. 
parcela foi composta de três fileiras com cinco plantas de cada cultivar, espaçadas 3,0 x 2,0 m, correspondendo à densidade de 1.666 plantas ha-1 ${ }^{-1}$ Considerou-se útil a fileira central, exceto as plantas das extremidades.

O preparo do solo constou de aração, gradagem e abertura de covas de plantio de 50 x 50 x $50 \mathrm{~cm}$, com auxílio de enxada, adicionando-se $5 \mathrm{~kg}$ de esterco de ave curtido por cova. As adubações foram realizadas com base na análise química do solo e recomendações para a cultura da banana (Alves, 1999). As adubações de manutenção foram feitas via água de irrigação, de acordo com a necessidade nutricional da cultura, baseando-se nos resultados da análise de solo. Durante o primeiro mês realizaram-se irrigações com água de $0,55 \mathrm{dS} \mathrm{m}^{-1}$; em seguida, iniciaram-se os tratamentos com água salina, irrigando-se com $15 \mathrm{~L} \mathrm{cova}^{-1}$ a cada dois dias no segundo mês; a partir do terceiro mês, as irrigações foram diárias com a lâmina de irrigação calculada a partir da evapotranspiração da cultura calculada conforme Allen et al. (1996), acrescida da lâmina de lixiviação correspondente a 15\%. A umidade do solo foi monitorada por tensiômetros instalados em oito subparcelas, uma para cada tratamento, nas profundidades de 15, 30 e $45 \mathrm{~cm}$. Durante o período chuvoso as irrigações suplementares foram realizadas com água de $0,5 \mathrm{dS} \mathrm{m}^{-1}$.

O sistema de irrigação utilizado foi o de gotejamento, com duas linhas laterais por fileira e quatro gotejadores por planta, com vazão de $3,75 \mathrm{~L} \mathrm{~h}^{-1}$ cada. Os níveis de salinidade foram obtidos a partir de misturas de água natural de poços do arenito $\left(\mathrm{CE}=0,55 \mathrm{dS} \mathrm{m}^{-1}\right.$ ) e do aqüífero calcário $\left(\mathrm{CE}=4,0 \mathrm{dS} \mathrm{m}^{-1}\right)$, colocando-se metade da água de 0,55 em 4,0 dS m ${ }^{-1}$, para obtenção da água de condutividade elétrica de 2,8 dS m-1; já para obtenção da água com condutividade elétrica de $1,7 \mathrm{dS} \mathrm{m}^{-1}$, foram misturadas, em partes iguais, as águas de 2,8 e $0,55 \mathrm{dS} \mathrm{m}^{-1}$, armazenadas em tanques de alvenaria revestidos de cimento, em que os níveis de salinidade foram verificados diariamente, com o auxílio de condutivímetro.

Realizou-se a colheita aos dez meses do segundo ciclo adotando-se, como critério de ponto de colheita, a mudança de tonalidade da casca, de verde-escuro para verde-claro. Por ocasião da colheita procedeu-se à avaliação das seguintes características: massa fresca do cacho; número de pencas e frutos por cacho; comprimento do fruto, obtido pela média dos comprimentos de dez frutos representativos por cacho por repetição; massa média do fruto; diâmetro do fruto e da polpa e produtividade.

Os dados obtidos foram analisados utilizando-se do Sistema de Análises Estatísticas e Genéticas da UFV (SAEG), sendo submetidos às análises de variância. Para comparar a média entre os tratamentos utilizou-se o teste de Tukey adotando-se o nível de 5\% de probabilidade.

\section{RESULTADOS E DISCUSSÃO}

Não se constatou efeito significativo da interação entre níveis de salinidade da água de irrigação e as cultivares de banana em relação às características avaliadas, indicando que as cultivares se comportaram de maneira semelhante quanto aos níveis de salinidade utilizados (Tabela 2); tampouco, se constatou efeito significativo dos níveis de salinidade da água de irrigação sobre o diâmetro da polpa (DP), diâmetro do fruto (DF), comprimento do fruto (CF), massa média da penca (MMP), massa total dos frutos (MTF) e massa fresca do engaço (MEN); as variedades não se diferenciaram em diâmetro da polpa (DP), massa fresca do engaço (MEN), número de pencas por cacho $(\mathrm{PCH})$ e número de frutos por cacho (FCH).

Esses resultados foram semelhantes aos obtidos com banana cultivar Nanica do grupo Cavendish, por Israeli et al. (1986) no primeiro ano de cultivo, quando verificaram que os níveis de salinidade de 1,25, 3,03, 4,25 e 4,34 dS m-1 não influenciaram o número de pencas nem de frutos por cacho; já no segundo cultivo foi significativa a influência dos níveis de salinidade $1,09,3,61,6,02$ e $5,86 \mathrm{dS} \mathrm{m}^{-1}$ para as características, número de pencas e frutos por cacho e massa do cacho, apresentando decréscimo para a massa do cacho, em torno de $18 \%$ no primeiro cultivo e de $60 \%$ no segundo cultivo, em decorrência do maior tempo de acúmulo de sais no solo e dos conseqüentes efeitos sobre os processos fisiológicos na planta.

Em condições normais, sem estresse salino, Scarpare Filho \& Kluge (2001) obtiveram 23,4 kg para a massa do cacho, 39,0 $\mathrm{t} \mathrm{ha}^{-1}$ para produtividade, $161,5 \mathrm{~g}$ para a massa média dos frutos, $21,5 \mathrm{~cm}$ para o comprimento dos frutos. Pereira et al. (2000), também encontraram, nas mesmas condições,

Tabela 2. Resumo da análise de variância das variáveis diâmetro da polpa (DP), diâmetro do fruto (DF), comprimento do fruto (CF), massa média da penca (MMP), massa total dos frutos (MTF), massa total das pencas (MTP), massa do cacho (MCH), massa média dos frutos (MMF), massa do engaço (MEN), número de pencas por cacho $(\mathrm{PCH})$, número de frutos por cacho $(\mathrm{FCH})$, e produtividade (PROD) de banana.

\begin{tabular}{|c|c|c|c|c|c|c|c|c|c|c|c|c|c|}
\hline Fonte de variação & GL & DP & DF & CF & MMP & MTF & MTP & $\mathrm{MCH}$ & MMF & MEN & $\mathrm{PCH}$ & $\mathrm{FCH}$ & PROD \\
\hline Bloco & 3 & $0,24^{\mathrm{ns}}$ & $1,39^{\text {ns }}$ & $1,20^{\mathrm{ns}}$ & $4,66^{*}$ & $1,22^{\mathrm{ns}}$ & $7,98^{\star \star}$ & $8,46^{\star *}$ & $5,20^{*}$ & $2,39^{\text {ns }}$ & $1,23^{\text {ns }}$ & $2,59^{\mathrm{ns}}$ & $8,46^{* *}$ \\
\hline Salinidade & 2 & $0,79^{\text {ns }}$ & $0,69^{\text {ns }}$ & $0,27^{\text {ns }}$ & $2,34^{\mathrm{ns}}$ & $0,97^{\mathrm{ns}}$ & $9,43^{* *}$ & $9,09^{* *}$ & $8,17^{\star *}$ & $0,77^{\mathrm{ns}}$ & $7,05^{*}$ & $4,61^{*}$ & $9,09^{\star *}$ \\
\hline QMRes (a) & 6 & 0,234 & 0,165 & 2,827 & 34297,97 & 2645852,0 & 0,622 & 0,616 & 205,218 & 0,031 & 0,048 & 8,489 & 1,712 \\
\hline Cultivar & 1 & $5,01^{\mathrm{ns}}$ & $10,38^{*}$ & $6,94^{*}$ & $21,93^{\star *}$ & $9,24^{*}$ & $20,57^{\star *}$ & $20,16^{* *}$ & $28,46^{* *}$ & $0,16^{\mathrm{ns}}$ & $0,23^{\text {ns }}$ & $2,63^{\text {ns }}$ & $20,16^{* \star}$ \\
\hline Cult $x$ Sal & 2 & $0,08^{\text {ns }}$ & $0,20^{\text {ns }}$ & $0,03^{\text {ns }}$ & $0,02^{\text {ns }}$ & $0,16^{\text {ns }}$ & $0,23^{\text {ns }}$ & $0,11^{\mathrm{ns}}$ & $0,22^{\mathrm{ns}}$ & $0,73^{\text {ns }}$ & $0,52^{\text {ns }}$ & $1,58^{\mathrm{ns}}$ & $0,11^{\mathrm{ns}}$ \\
\hline C.V(\%) (b) & & 18,01 & 13,51 & 12,69 & 22,77 & 32,55 & 22,08 & 18,83 & 20,36 & 6,27 & 6,46 & 5,43 & 18,83 \\
\hline
\end{tabular}

**, , , ns: Significativo a 1 e 5 \% de probabilidade, e Não significativo pelo teste "F", respectivamente 
Tabela 3. Diâmetro da polpa (DP), diâmetro do fruto (DF), comprimento do fruto (CF), massa média da penca (MMP), massa total dos frutos (MTF), massa total das pencas (MTP), massa do cacho $(\mathrm{MCH})$, massa média dos frutos (MMF), massa engaço (MEN), número de pencas por cacho (PCH), número de frutos por cacho (FCH), e produtividade (PROD) de banana em função das cultivares 'Marmelo' e 'Pacovan'.

\begin{tabular}{|c|c|c|c|c|c|c|c|c|c|c|c|c|}
\hline Cultivar & $\begin{array}{l}\text { DP } \\
\text { (cm) }\end{array}$ & $\begin{array}{l}\text { DF } \\
\text { (cm) }\end{array}$ & $\begin{array}{l}\text { CF } \\
(\mathrm{cm})\end{array}$ & $\begin{array}{c}\text { MMP } \\
(\mathrm{kg})\end{array}$ & $\begin{array}{l}\text { MTF } \\
(\mathrm{kg})\end{array}$ & $\begin{array}{l}\text { MTP } \\
(\mathrm{kg})\end{array}$ & $\begin{array}{l}\mathrm{MCH} \\
(\mathrm{kg})\end{array}$ & $\begin{array}{c}\text { MMF } \\
\text { (g) }\end{array}$ & $\begin{array}{l}\text { MEN } \\
(\mathrm{kg})\end{array}$ & PCH & $\mathrm{FCH}$ & $\begin{array}{l}\text { PROD } \\
\left(\mathrm{t} \mathrm{ha}^{-1}\right)\end{array}$ \\
\hline Marmelo & $3,63 a^{*}$ & $4,36 \mathrm{a}$ & $16,01 a$ & $1,66 \mathrm{a}$ & $9,11 \mathrm{a}$ & $8,70 \mathrm{a}$ & $9.99 \mathrm{a}$ & $150,66 \mathrm{a}$ & $1,28 \mathrm{a}$ & $5,33 \mathrm{a}$ & $57,74 a$ & $16,64 \mathrm{a}$ \\
\hline Pacovan & $3,08 \mathrm{a}$ & $3,64 \mathrm{~b}$ & $13,97 \mathrm{~b}$ & $1,06 \mathrm{~b}$ & $6,05 \mathrm{~b}$ & $5,74 \mathrm{~b}$ & $7,05 \mathrm{~b}$ & $95,97 \mathrm{~b}$ & $1,30 \mathrm{a}$ & $5,40 \mathrm{a}$ & $59,85 \mathrm{a}$ & $11,74 b$ \\
\hline
\end{tabular}

"Médias seguidas da mesma letra na coluna não diferem entre si $(p>0,05)$ pelo teste de Tukey

Tabela 4. Diâmetro da polpa (DP), diâmetro do fruto (DF), comprimento do fruto (CF), massa média da penca (MMP), massa total dos frutos (MTF), massa total das pencas (MTP), massa do cacho $(\mathrm{MCH})$, massa média dos frutos (MMF), massa engaço (MEN), número de pencas por cacho (PCH), número de frutos por cacho $(\mathrm{FCH})$, e produtividade (PROD) de banana em função dos níveis de salinidade da água de irrigação.

\begin{tabular}{|c|c|c|c|c|c|c|c|c|c|c|c|c|}
\hline Salinidade de água & $\begin{array}{c}\text { DP } \\
\text { (cm) }\end{array}$ & $\begin{array}{c}\mathrm{DF} \\
(\mathrm{mm})\end{array}$ & $\begin{array}{l}\text { CF } \\
(\mathrm{cm})\end{array}$ & $\begin{array}{c}\text { MMP } \\
(\mathrm{kg})\end{array}$ & $\begin{array}{l}\text { MTF } \\
(\mathbf{k g})\end{array}$ & $\begin{array}{l}\text { MTP } \\
(\mathrm{kg})\end{array}$ & $\begin{array}{c}\mathrm{MCH} \\
(\mathrm{kg})\end{array}$ & $\begin{array}{c}\text { MMF } \\
\text { (g) }\end{array}$ & $\begin{array}{l}\text { MEN } \\
(\mathbf{k g})\end{array}$ & PCH & $\mathrm{FCH}$ & $\begin{array}{l}\text { PROD } \\
\left(t \mathrm{ha}^{-1}\right)\end{array}$ \\
\hline $1,70 \mathrm{dS} \mathrm{m}^{-1}$ & $3,19 a^{*}$ & $3,88 \mathrm{a}$ & $14,63 \mathrm{a}$ & $1,43 \mathrm{a}$ & $7,18 \mathrm{a}$ & $8,04 \mathrm{a}$ & $9,35 \mathrm{a}$ & $132,7 \mathrm{a}$ & $1,31 \mathrm{a}$ & $5,60 \mathrm{a}$ & $60,61 \mathrm{a}$ & $15,58 \mathrm{a}$ \\
\hline $2,80 \mathrm{dS} \mathrm{m}^{-1}$ & $3,38 \mathrm{a}$ & $4,00 \mathrm{a}$ & $15,15 \mathrm{a}$ & $1,41 \mathrm{a}$ & $7,33 \mathrm{a}$ & $7,28 \mathrm{ab}$ & $8,52 \mathrm{ab}$ & $130,5 \mathrm{a}$ & $1,23 \mathrm{a}$ & $5,22 \mathrm{~b}$ & $56,33 \mathrm{~b}$ & $14,20 a b$ \\
\hline $4,00 \mathrm{dS} \mathrm{m}^{-1}$ & $3,50 \mathrm{a}$ & $4,12 \mathrm{a}$ & $15,19 \mathrm{a}$ & $1,25 \mathrm{a}$ & $8,23 \mathrm{a}$ & $6,33 \mathrm{~b}$ & $7,68 \mathrm{~b}$ & $106,6 \mathrm{~b}$ & $1,34 \mathrm{a}$ & $5,27 \mathrm{~b}$ & $59,44 a b$ & $12,79 \mathrm{~b}$ \\
\hline
\end{tabular}

* Médias seguidas da mesma letra na coluna não diferem entre si $(p>0,05)$ pelo teste de Tukey

resultados de $17,7 \mathrm{~kg}$ para a massa do cacho, 29,1 t ha-1 para produtividade, $150,0 \mathrm{~g}$ para a massa média dos frutos, $22,5 \mathrm{~cm}$ para o comprimento dos frutos em média. Todos esses valores foram elevados, comparados com os obtidos neste trabalho, que foram de 9,99 kg, 16,64 t ha-1, 150,6 g e 16,0 cm, respectivamente, mas se salienta que esta diferença pode estar relacionada às cultivares e aos níveis de salinidade de água de irrigação utilizados no presente estudo (Tabela 3).

De maneira geral, a cultivar 'Marmelo' apresentou melhor desempenho do que a 'Pacovan' (Tabela 3). Esses resultados indicam que a cultivar 'Marmelo' apresenta maior potencial produtivo que a 'Pacovan', independente dos níveis de salinidade aplicados. Este comportamento pode ser atribuído às diferenças genéticas inerentes a cada genótipo, como verificado pela maior massa fresca média do cacho $(9,99 \mathrm{~kg})$, representando uma produtividade de 16,64 tha-1, considerando-se a densidade de plantio de 1.666 plantas ha ${ }^{-1}$. Por se tratar de cultivos irrigados com água salina, pode-se considerar que o desempenho foi adequado, uma vez que a produtividade média nacional tem sido inferior a $16 \mathrm{t} \mathrm{ha}^{-1}$ (FNP - Consultoria \& Comércio, 2005).

Constatou-se efeito significativo dos níveis de salinidade para massa total das pencas, massa do cacho, massa média dos frutos, número de pencas por cacho, número de frutos por cacho e produtividade (Tabela 4). Para todas as características, verificou-se redução na performance com o aumento na concentração salina (1,7 para $\left.4,0 \mathrm{dS} \mathrm{m}^{-1}\right)$ da água de irrigação. A redução foi de 21,27\% para massa total das pencas, $17,90 \%$ para massa do cacho, $19,67 \%$ para a massa média dos frutos, 5,89\% para o número de pencas por cacho, 1,93\% para o número de frutos por cacho, e 17,90\% para a produtividade. Israeli et al. (1986) notaram que a produtividade foi significativamente afetada pela qualidade da água de irrigação, havendo um declínio evidente com níveis em torno de 3,6 dS m-1. Abreu et al. (1982), também constataram redução de $40 \%$ na produtividade de banana, com o aumento da salinidade. O estresse salino, tem reduzi- do de modo geral tanto o crescimento quanto a produtividade das plantas, foi o que se observou por Gondim et al. (2002) e Carmo et al. (2003).

Em geral, o efeito tóxico do Na também está presente e é um complicador a mais na salinidade, seu efeito tóxico provoca redução na absorção e no equilíbrio dos cátions (K, Mg e Ca) e mudanças no metabolismo da planta, que ocasionam redução no seu crescimento e produção (Marschner, 1995).

\section{CONCLUSÕES}

1. O aumento da condutividade eletríca da água de irrigação de 1,7 para $4,0 \mathrm{dS} \mathrm{m}^{-1}$, diminuiu em 21,27, 17,90, $19,67,5,89,1,93$ e $17,90 \%$, respectivamente, a massa total das pencas, massa do cacho, massa média dos frutos, número de pencas por cacho, número de frutos por cacho e produtividade.

2. Dentre os níveis de salinidade da água de irrigação testados, o menor nível estudado $\left(1,7 \mathrm{dS} \mathrm{m}^{-1}\right)$ proporcionou produção satisfatória.

3. A cultivar 'Marmelo' indicou maior potencial produtivo em relação à 'Pacovan'.

4. O efeito da salinidade nas cultivares foi semelhante.

\section{LITERATURA CITADA}

Abreu, H. J. M.; Mascrell, J.; Duart, S.; Socorro, A. R. Na and $\mathrm{Cl}$ content in banana plants of the Canaria Islands. International Banana Nutrition Newsletter, Nedlands, v.15, n.5, p.1314, 1982.

Allen, R. G.; Smith, M.; Pereira, L. S.; Pruit, W.O. Proposed revision to the FAO procedure for estimating crop water requirements. In: International Symposium on Irrigation of Horticultural Crops, 2, 1996, Chania. Proceedings... Leuven: ISHS, 1996. v.1, p. 17-33. 
Alves, J. E. A cultura da banana: aspectos técnicos sócioeconômicos e agroindustriais. 2. ed., revisada, Brasília: Embrapa SPI, 1999, 586p.

Araújo Filho, J. B. de; Gheyi, H.R.; Azevedo, N.C. de. Tolerância da bananeira à salinidade em fase inicial de desenvolvimento. Pesquisa Agropecuária Brasileira, Brasília, v.30, n.7, p.989997, 1995.

Bernardo, S. Manual de irrigação. 6.ed. Viçosa: UFV, 1995. 657p.

Carmo, G. A.; Medeiros, J. F.; Tavares, J. C.; Gheyi, H.R.; Souza, A. M.; Palácio, E. A. Q. Crescimento de bananeiras sob diferentes níveis de salinidade da água de irrigação. Revista Brasileira de Fruticultura, Jaboticabal, v.25, n.3, p.513-518, 2003.

Costa, R. G.; Gheyi, H.R. Variação da água de irrigação da microregião homogênea de Catolé do Rocha, $\mathrm{PB}$. Pesquisa Agropecuária Brasileira, Brasília, v.19, n.8, p.1021-1025, 1984.

Drew, M. C.; Hole, P.S.; Picchioni, G. A. Inhibition by $\mathrm{NaCl}$ of net $\mathrm{CO}_{2}$ fixation and yield of cucumber. Journal of the American Society for Horticultural Science, Alexandria, v.115, n.3, p.472-477, 1990.

EMBRAPA, Empresa Brasileira de Pesquisa Agropecuária - Sistema brasileiro de classificação de solos. Brasília: Serviço de Produção de Informação, 412p, 1999.

FAO. Water, soil and crop management relating the use of saline water. AGL/MISC/16/90, Rome. 1990.http://www.apps.fao.org. 15 Ago.2004.

FNP - Consultoria \& Comércio, AGRIANUAL 2005. Anuário da agricultura brasileira. São Paulo:IFNP. 2005, 169p.

Gondim, A. R. O.; Medeiros, J. F.; Levien, S. L. A.; Carmo, G. A. Coeficiente de cultura e salinidade do solo na fase reprodutiva da bananeira irrigada com diferentes níveis de salinidade. In: Congresso Brasileiro de Engenharia Agrícola, 31, 2002, Salvador, Anais..., Salvador: SBEA, 2002. CD-Rom.

Israeli, Y.; Lahav, E.; Nameri, N. The effect of salinity and sodium adsorption ratio in the irrigation water, on growth and productivity of bananas under irrigation conditions. Fruits, Paris, v.41, n.5, p.297-302, 1986.

Maia, C. E.; Morais, E. R. C.; Oliveira, M. Qualidade da água para fertirrigação por gotejamento. I: região da Chapada do ApodíRN. Engenharia na Agricultura, Viçosa, v.5, p.281-292, 1997.
Marschner, H. Mineral nutrition of higher plants. London: Academic Press, 1995. 889p.

Medeiros, J. F. de; Gheyi, H. R.; Batista, M. A. F. Procedimentos de análise de solo e água para diagnóstico de salinidade. Mossoró: ENA, ESAM, 1993. 25p. Coleção Mossoroense, Série E, 1256

Meloni, D. A., Oliva, M. A., Ruiz, H. A., Martinez, C. A. Contribution of proline and inorganic solutes to osmotic adjustment in cotton under salt stress. Journal of Plant Nutrition, Atenas, v.24, n.3, p.599-612, 2001.

Neves, L. L. de M.; Siqueira, D. L. de; Cecon, P. R.; Martinez, C. A.; Salomão, L. C. C. Crescimento, trocas gasosas e potencial osmótico da bananeira-'prata', submetida a diferentes doses de sódio e cálcio em solução nutritiva. Revista Brasileira de Fruticultura, Jaboticabal, v.24, n.2, p.524-529, 2002.

Pereira, F. H. F.; Espinula Neto, D.; Soares, D. C.; Oliva, M. A. Trocas gasosas em plantas de tomateiro submetidas a condições salinas. Horticultura Brasileira, Campo Grande, v.22, n.2, 2004. CD-Rom

Pereira, M. C. T.; Salomão, L. C. C.; Silva, S. de O.; Sediyama, C. S.; Couto, F. A. D.; Silva Neto, S. P. Crescimento e produção de primeiro ciclo da bananeira 'Prata Anã' (AAB) em sete espaçamentos. Pesquisa Agropecuária Brasileira, Brasília, v.35, n.7, p.1377-1387, 2000.

Santos, J. G. R. dos; Gheyi, H. R. Efeito da salinidade da água na composição da folha da bananeira e nas características do solo. Pesquisa Agropecuária Brasileira, Brasília, v.29, n.2, p.247253, 1994.

Scarpare Filho, J. A. E.; Kluge R. A. Produção da bananeira 'Nanicão’ em diferentes densidades de plantas e sistemas de espaçamento. Pesquisa Agropecuária Brasileira, Brasília, v.36, n.1, p.105-113, 2001.

Silva, J. T. A.; Borges, A. L.; Malburg, J. L. Solos, adubação e nutrição da bananeira. Informe Agropecuário, Belo Horizonte, v.6, n.63, p.18-21, 1999.

Ulisses, C.; Câmara, T. R.; Willadino, L.; Meunier, I.; Sérgio, P.; Rocha, G. da; Albuquerque, C. Seleção in vitro de gemas de bananeira 'nanicão' tolerantes à salinidade. Scientia Agricola, Piracicaba, v.57, n.4, p.667-670, 2000. 